\title{
Imaging-based outcome prediction in posterior circulation stroke
}

\author{
Helge C. Kniep ${ }^{1}$ - Sarah Elsayed ${ }^{1}$. Jawed Nawabi ${ }^{1,2}$. Gabriel Broocks ${ }^{1} \cdot$ Lukas Meyer $^{1} \cdot$ Matthias Bechstein $^{1}$. \\ Noel Van Horn ${ }^{1} \cdot$ Marios Psychogios ${ }^{4}$. Götz Thomalla ${ }^{5}$. Fabian Flottmann ${ }^{1}$. André Kemmling ${ }^{3}$. Susanne Gellißen ${ }^{1}$. \\ Jens Fiehler ${ }^{1}$ Peter B. Sporns ${ }^{1,4}$. Uta Hanning ${ }^{1}$
}

Received: 30 October 2021 / Revised: 3 February 2022 / Accepted: 3 February 2022 / Published online: 7 March 2022

(c) The Author(s) 2022

\begin{abstract}
Background and purpose We developed a machine learning model to allow early functional outcome prediction for patients presenting with posterior circulation (pc)-stroke based on CT-imaging and clinical data at admission. The proposed algorithm utilizes quantitative information from automated multidimensional assessments of posterior circulation Acute Stroke Prognosis Early CT-Score (pc-ASPECTS) regions. Discriminatory power was compared to predictions based on conventional pc-ASPECTS ratings.

Methods We retrospectively analyzed non-contrast CTs and clinical data of 172 pc-stroke patients. 90 days outcome was dichotomized into good and poor using modified Rankin Scale (mRS) cut-offs. Predictive performance was assessed for outcome differentiation at $\mathrm{mRS} 2,3,4$ and survival prediction $(\mathrm{mRS} \leq 5)$ using random forest algorithms. Results were compared to conventional pc-ASPECTS and clinical parameters. Models were evaluated in a nested fivefold cross-validation approach. Results Receiver operating characteristic areas under the curves (ROC-AUCs) of the test sets using conventionally rated pc-ASPECTS reached 0.63 for $\mathrm{mRS} \leq 4$ to 0.68 for $\mathrm{mRS} \leq 5$ and 0.73 for $\mathrm{mRS} \leq 5$ to 0.85 for $\mathrm{mRS} \leq 2$ if clinical data were considered. Pure imaging-based machine learning classifier ROC-AUCs were lowest for $\mathrm{mRS} \leq 4(0.81)$ and highest for $\mathrm{mRS} \leq 5$ (0.87). The combined clinical data and machine learning-based model had the highest predictive performance with ROC-AUCs reaching 0.90 for $\mathrm{mRS} \leq 2$.

Conclusion Machine learning-based evaluation of pc-ASPECTS regions predicts functional outcome of pc-stroke patients with higher accuracy than conventional assessments. This could optimize triage for additional diagnostics and allocation of best possible medical care and might allow required arrangements of the social environment at an early point of time.
\end{abstract}

Keywords Posterior circulation stroke $\cdot$ Outcome prediction $\cdot$ Machine learning $\cdot$ Pc-ASPECTS

Helge C. Kniep, Sarah Elsayed, Peter B. Sporns and Uta Hanning contributed equally to the manuscript.

Helge C. Kniep

h.kniep@uke.de

1 Department of Diagnostic and Interventional Neuroradiology, University Medical Center HamburgEppendorf, Hamburg, Germany

2 Department of Radiology, Charité School of Medicine and University Hospital Berlin, Berlin, Germany

3 Department of Neuroradiology, University Medical Center Marburg, Marburg, Germany

4 Department of Diagnostic and Interventional Neuroradiology, Clinic for Radiology and Nuclear Medicine, University Hospital Basel, Basel, Switzerland

5 Department of Neurology, University Medical Center Hamburg-Eppendorf, Hamburg, Germany

\section{Introduction}

Posterior circulation (pc) strokes are frequently associated with poor outcome $[8,17]$. Recently reported results from the Basilar artery international cooperation study (BASICS) indicate that functional outcome may still, to a large extent, be dependent on the initial clinical presentation and imaging findings, and, to a lesser extent, on the specific therapeutic strategies [14, 32]. Predicting functional outcome based on the initial clinical and imaging findings might therefore (1) allow for a prognosis of the patient's long-term functional status (2) optimize triage for additional MR imaging diagnostics and allocation of best possible medical care [36] and (3) facilitate required adaptations in the patient's social environment including arrangements of long-term care at an early point of time. 
Binary quantification of early ischemic changes using the posterior circulation Acute Stroke Prognosis Early CT Score (pc-ASPECTS) was shown to predict functional outcome in patients with suspected pc ischemia [27, 30]. However, conventional binary classifications of pc-ASPECTS regions do not consider all information available from the imaging data: prognostic value carried by changes in texture and small shifts of grey level distributions remains unused. The accuracy of conventional pc-ASPECTS ratings is also affected by the limited sensitivity of the human eye for subtle early ischemic changes. Moreover, visual assessments of non-contrast CT (NCCT) images suffers from inter- and intra-reader variability and are often interfered by beamhardening artifacts in the posterior fossa [9, 27, 33].

The integration of clinical data, mainly the baseline National Institute of Health Stroke Scale (NIHSS) was shown to improve discriminatory power [16]. However, although being the most widely used scoring system in patients with acute ischemic stroke, NIHSS has weaknesses when applied to pc strokes partly because deficits such as truncal ataxia, dysphagia and diplopia—that are typical for pc strokes-are not assessed. This explains why patients with pc stroke can have a high probability of an unfavorable outcome at 90 days despite relatively low NIHSS scores at admission [31] and underlines the need of a combined approach of imaging evaluation and clinical scoring [16].

We therefore propose a machine learning (ML)-based evaluation of multidimensional quantitative image features from pc-ASPECTS regions in admission NCCTs combined with clinical data to predict functional outcomes in patients with acute pc strokes.

\section{Materials and methods}

The anonymized data used for training and validation of algorithms that support the findings of this study are available from the corresponding author upon reasonable request.

This multi-center retrospective study was approved by the Ethics Committee of the University of Hamburg and the Hamburg Chamber of Physicians, Hamburg, Germany, and the Ethics Committee of the University of Muenster and the Westfalian Chamber of Physicians, Muenster, Germany, and written informed consent was waived by the institutional review boards. All study protocols and procedures were conducted in accordance with the Declaration of Helsinki.

\section{Patient characteristics}

The study cohort includes consecutive patients with suspected posterior circulation ischemia admitted between April 1, 2010, and February 28, 2019 at two tertiary care stroke centers. Inclusion criteria for this study were (1) documented occlusion of the basilar or intracranial vertebral artery; (2) NCCT performed on admission within $6 \mathrm{~h}$ of symptom onset; (3) availability of modified Rankin Scale (mRS) after 90 days (mRS90). Patients were excluded in case of poor imaging quality (artifacts from movement and implants). In total, 172 patients met the inclusion criteria and were selected for the imaging-based analysis. Complete clinical data including NIHSS at admission were available for 149 patients that were selected for all models employing clinical data at admission.

\section{Image acquisition}

NCCT scans with head images obtained from the vertex to the skull base were acquired on a 128-slice dual-source CT scanner (Somatom Definition Flash; Siemens Healthcare $\mathrm{GmbH}$ ) with tube voltage $120 \mathrm{kV}$, tube current $340 \mathrm{~mA}$, $5.0 \mathrm{~mm}$ slice reconstruction, $<0.5 \mathrm{~mm}$ in-plane resolution, as well as on an iCT $256^{\mathrm{TM}}$ scanner (Philips Healthcare, Best, The Netherlands) with tube voltage $120 \mathrm{kV}$, tube current $300 \mathrm{~mA}, 4.0 \mathrm{~mm}$ slice reconstruction and $<0.5 \mathrm{~mm}$ inplane resolution.

\section{Visual pc-ASPECTS rating}

For all admission NCCT scans, pc-ASPECTS was conventionally assessed by two Neuroradiologists in a consensus rating approach (UH, PS: 8 years of clinical experience in diagnostic neuroradiology in acute care full-service hospitals). pc-ASPECTS allots the posterior circulation 10 points. One point each is subtracted for early ischemic changes on NCCT in left or right thalamus, cerebellum, or posterior cerebral artery territory, respectively, and two points each for early ischemic changes in any part of the midbrain or pons. A pc-ASPECTS score of 10 indicates absence of visible posterior circulation ischemia, a score of 0 indicates early ischemic changes in all pc-ASPECTS territories [7, 27].

\section{Image pre-processing and pc-ASPECTS feature extraction}

To (1) extract information from standardized pc-ASPECTS maps and (2) reduce potential bias in quantitative texture analysis, all NCCT images were registered to a custom MNI (Montreal Neurological Institute)-152 CT reference atlas [10] using two-step affine algorithms. Registration success was visually verified by two Neuroradiologists (UH, PS). Standardized pc-ASPECTS area maps (thalamus left/right $(1 / r)$, pons, midbrain, territory of the posterior cerebral artery (PCA) 1/r, cerebellum 1/r) were derived as follows: First, an experienced Neuroradiologist (UH) performed manual segmentations of the respective regions on the original NCCT images of 63 healthy subjects using Analyze 11.0 Software 
(Biomedical Imaging Resource, Mayo Clinic, Rochester, MN) [3]. Second, manual segmentations were transformed into standard space by utilizing transformation matrices obtained from image registration to the custom MNI-152 CT reference atlas [10]. Third, all segmentations were added and final standard maps were defined by applying $50 \%$ cutoff points.

Quantitative image features were extracted using the PyRadiomics Python package v2.1.0 [35], proposed default settings were used for the analysis. Extracted features comprised 252 first-order features (18 based on unfiltered images, 144 wavelet decompositions, 90 log-sigma Laplacian of Gaussian filtered images) and 966 texture features (82 based on unfiltered images, 544 wavelet decompositions, 340 log-sigma Laplacian of Gaussian filtered images). In total, 1218 quantitative image features were extracted from each of the 1376 included pc-ASPECTS areas.

\section{Statistical analysis}

Univariate logistic regression analysis was conducted based on the entire dataset to investigate conventional odds ratios of the clinical predictors (NIHSS at admission, pc-ASPECTS and age) for good outcome (mRS90 $\leq 2)$. Using fivefold cross validation, univariate (conventional pc-ASPECTS ratings) and multivariate logistic regression models (conventional pc-ASPECTS ratings, NIHSS at admission and age) were trained to predict functional outcome at dichotomized mRS90 levels of $\leq 2, \leq 3, \leq 4$ and $\leq 5$ (survival).

Imaging-based machine learning prediction of dichotomized mRS90 levels was evaluated using Random Forest algorithms (Python scikit-learn environment v0.20.3 [24]) in a fivefold nested cross validation approach [15]. Random forest classifiers have a comparably low tendency to overfit [4] and support classification tasks also for data sets comprising numerous and heterogeneous predictors. For each study patient, quantitative image features of the eight pcASPECTS regions were evaluated for their ability to predict functional outcome (9744 image feature in total per patient). Hyperparameter tuning of the random forest classifiers (total number of features, number of trees, maximum depth of the tree, minimum number of samples to split an internal node, number of features considered for splitting $\left(m_{\text {try }}\right)$, minimum number of samples at leaf node) was conducted using grid search algorithms on each training data set within the nested cross-validation layers. Parameters at initiation were set to scikit-learn default values. Selection of features with the highest predictive value was conducted separately for each training data set of the fivefold cross-validation sample split according to Gini impurity measures [18]. For the integrated model, predicted probabilities for good outcome of the logistic regression model using clinical data and of the imagingbased machine learning classifier were averaged.
Receiver operating characteristic (ROC) curves were used to determine the optimal cut-off values according to Youden's index. For predictive models, ROC curves were generated from results of all cross-validation sets. Confidence intervals (CI) for sensitivities and specificities were bootstrapped (2000 replicates, pROC v1.15 R-package [29]). Bonferroni adjustments were applied to control for alpha error inflation. Furthermore, the classifiers were analyzed using sensitivity, specificity, accuracy, maximum Youden Index, positive predictive value, negative predictive value (ThresholdROC v2.8 R-package) and Matthews correlation coefficient (MCC) [20] metrics (psychometric v.2.2. R-package). MCC evaluates all fields of the confusion matrix and is considered as a favorable measure for unbiased comparisons of binary classifiers [25]. Due to the relatively low class imbalance for all mRS90 cut-off values (event rates for $m R S 90 \leq 2: 33 \% ; \leq 3: 40 \%$; $\leq 4: 56 \%$; $\leq 5: 74 \%$ ), no additional data augmentation for reducing bias from class imbalance was performed.

A graphical flow chart of the proposed ML-based algorithm for prediction of the clinical outcome is depicted in Fig. 1.

\section{Results}

Our analysis included NCCT images of 1376 pc-ASPECTS regions extracted from 172 patients ( 77 females, median age 74 years, interquartile range (IQR): 61-79 years) with acute stroke in the posterior circulation. NIHSS assessments were available for 149 patients. Median NIHSS score at admission was 15 (IQR 5-42), 94 patients (54.7\%) underwent successful recanalization with TICI (thrombolysis in cerebral infarctions) score $\geq 2 \mathrm{~b}, 79$ patients (45.9\%) were treated with intravenous thrombolysis (Table 1$) .57$ patients $(33.1 \%)$ reached a favorable outcome of $\mathrm{mRS} \leq 2$ at day 90 .

\section{Logistic regression analysis}

Logistic regression for $\mathrm{mRS} \leq 2$ (good outcome) of the conventional predictors (conventional pc-ASPECTS, NIHSS at admission and age) on the entire dataset showed significant coefficients for pc-ASPECTS and NIHSS at admission (P-value $<0.05)$, age was not significantly associated with good outcome (Table 2). Optimal cut-off values (Youden's index) indicate that patients with pc-ASPECTS $\geq 8$ have a significantly higher probability to achieve $\mathrm{mRS} \leq 2$ with odds ratio of 11.07 (95\% CI [2.55; 48.02]). Also patients with NIHSS at admission $<10$ have a significantly higher chance of good outcome with odds ratio of 16.17 (95\% CI [7.01; 37.32]). 


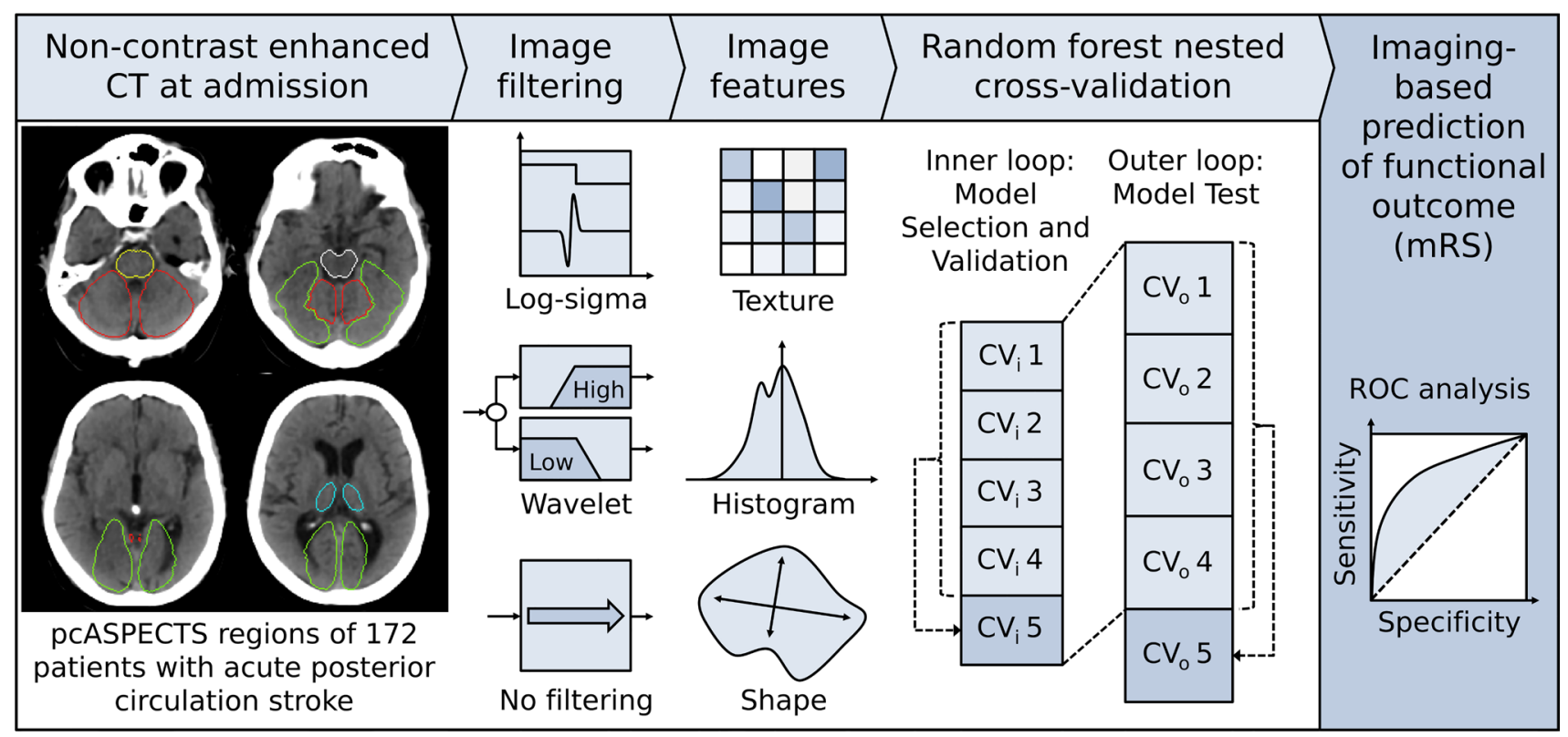

Fig. 1 Schematic overview of proposed imaging-based outcome prediction pipeline. $C V$ cross validation set, $m R S$ modified Rankin Scale, $p c$ ASPECTS posterior circulation Acute Stroke Prognosis Early CT Score, ROC receiver-operating-characteristic

Table 1 Patient characteristics

\begin{tabular}{|c|c|c|c|c|}
\hline & $\begin{array}{l}\text { Total number of } \\
\text { patients } n=172\end{array}$ & $\begin{array}{l}\text { mRS at } 90 \text { days } \leq 2 \\
n=57(33 \%)\end{array}$ & $\begin{array}{l}\mathrm{mRS} \text { at } 90 \text { days }>2 \\
n=115(67 \%)\end{array}$ & $p$ value \\
\hline Age in years, median (IQR) & $74(61-79)$ & $72(60-78)$ & $74(62-80)$ & 0.271 \\
\hline Female sex, $n(\%)$ & $77(44.77 \%)$ & $19(33.33 \%)$ & $58(50.43 \%)$ & 0.031 \\
\hline Hypertension, $n(\%)$ & $122(70.93 \%)$ & $36(63.16 \%)$ & $86(74.78 \%)$ & 0.218 \\
\hline Diabetes mellitus, $n(\%)$ & $44(25.58 \%)$ & $13(22.81 \%)$ & $31(26.96 \%)$ & 0,691 \\
\hline Hyperlipidemia, $n(\%)$ & $46(26.74 \%)$ & $18(31.58 \%)$ & $28(24.35 \%)$ & 0.266 \\
\hline Atrial fibrillation, $n(\%)$ & $62(36.01 \%)$ & $22(38.60 \%)$ & $40(34.78 \%)$ & 0.512 \\
\hline Intravenous thrombolysis, $n(\%)$ & $79(45.93 \%)$ & $21(36.84 \%)$ & $58(50.43 \%)$ & 0.064 \\
\hline Mechanical thrombectomy mTICI $2 \mathrm{~b} / 3, n(\%)$ & $94(54.65 \%)$ & $27(47.37 \%)$ & $67(58.26 \%)$ & 0.001 \\
\hline Admission NIHSS, median (IQR) $n=23$ missings & $15(5-42)$ & $3(2-10)$ & $30(13-42)$ & $<0.001$ \\
\hline NIHSS at $24 \mathrm{~h}$, median (IQR) $n=23$ missings & $6(2-42)$ & $2(1-4)$ & $36(15-42)$ & $<0.001$ \\
\hline Discharge NIHSS, median (IQR) $n=23$ missings & $3(2-17)$ & $2(1-3)$ & $17(10-29)$ & $<0.001$ \\
\hline mRS at 90 days, median (IQR) & $4(1-6)$ & $1(0-1)$ & $5(4-6)$ & $<0.001$ \\
\hline pc-ASPECTS, median (IQR) & $9(8-10)$ & $9(9-10)$ & $9(7-9)$ & $<0.001$ \\
\hline TICI, median (IQR) & $3(2-3)$ & $3(2-3)$ & $2(2-3)$ & $<0.001$ \\
\hline Time from onset to imaging in hours, median (IQR) & $4(1.45-10)$ & $6(1.75-19)$ & $3.20(1.40-6)$ & 0.024 \\
\hline
\end{tabular}

Demographic and clinical characteristics of the study cohort

$I Q R$ interquartile range, $m R S$ modified Rankin Scale, NIHSS National Institute of Health Stroke Scale, $p c-A S P E C T S$ posterior circulation Acute Stroke Prognosis Early CT Score, TICI thrombolysis in cerebral infarctions

\section{Predictive models for functional outcome}

Areas under the receiver operating characteristic curves (ROC AUCs) of the test sets using conventionally rated pc-ASPECTS in an univariate logistic regression reached $0.63(95 \%$ CI $[0.59 ; 0.67])$ for $\mathrm{mRS} \leq 4$ to 0.68 (95\% CI
$[0.63 ; 0.72])$ for $m R S \leq 5$. Pure imaging-based machine learning classifier ROC AUCs were lowest for $\mathrm{mRS} \leq 4$ with $0.81(95 \%$ CI $[0.78 ; 0.84])$ and highest for $\mathrm{mRS} \leq 5$ with 0.87 (95\% CI [0.85; 0.90]). Employing multidimensional conventional predictors (conventional pc-ASPECTS, NIHSS at admission and age) yielded ROC AUCs of 0.73 
Table 2 Logistic regression of conventional predictors for $\mathrm{mRS} 90 \leq 2$ (good outcome)

\begin{tabular}{|c|c|c|c|c|c|c|}
\hline & $\begin{array}{l}\text { Regression coef- } \\
\text { ficient }\end{array}$ & Standard error & P-value & Odds ratio & $95 \%$ CI lower & $95 \%$ CI upper \\
\hline pc-ASPECTS & 0.48 & 0.14 & $<0.001$ & 1.62 & 1.23 & 2.14 \\
\hline Intercept & -4.87 & 1.27 & $<0.001$ & 0.01 & & \\
\hline pc-ASPECTS $\geq 8$ & 2.40 & 0.75 & $<0.001$ & 11.07 & 2.55 & 48.02 \\
\hline Intercept & -2.80 & 0.73 & $<0.001$ & 0.06 & & \\
\hline NIHSS at admission & -0.10 & 0.02 & $<0.001$ & 0.90 & 0.87 & 0.94 \\
\hline Intercept & 1.07 & 0.31 & $<0.001$ & 2.91 & & \\
\hline NIHSS at admission $<10$ & 2.78 & 0.43 & $<0.001$ & 16.17 & 7.01 & 37.32 \\
\hline Intercept & -1.71 & 0.28 & $<0.001$ & 0.18 & & \\
\hline Age & -0.01 & 0.01 & 0.38 & 0.99 & 0.97 & 1.01 \\
\hline Intercept & -0.04 & 0.77 & 0.96 & 0.96 & & \\
\hline
\end{tabular}

Logistic regression of conventional clinical information for mRS 90 days $\leq 2$ (good outcome). Optimal cut-off values were determined using Receiver-Operating-Characteristic curve analysis Youden's index. Results are based on 149 patients from 2 different centers.

$m R S$ modified Rankin Scale, NIHSS National Institute of Health Stroke Scale, pc-ASPECTS posterior circulation Acute Stroke Prognosis Early CT Score

$(95 \%$ CI $[0.69 ; 0.77])$ for $\mathrm{mRS} \leq 5$ (lowest) to $0.85(95 \%$ CI $[0.82 ; 0.88])$ for $m R S \leq 2$. Overall, highest predictive performance was observed for the combined clinical data and machine learning-based model with ROC AUCs of 0.83 $(95 \% \mathrm{CI}[0.80 ; 0.87])$ for $\mathrm{mRS} \leq 5$ (lowest) to $0.90(95 \%$
CI $[0.88 ; 0.92]$ ) for $\mathrm{mRS} \leq 2$ (highest) (Figs. 2 and 3 and Table 3). Results show that the predictive performance of machine learning-based evaluation of quantitative image features was higher compared to the predictive value of conventional pc-ASPECTS metrics ( $p$ values $<0.05)$. If
A

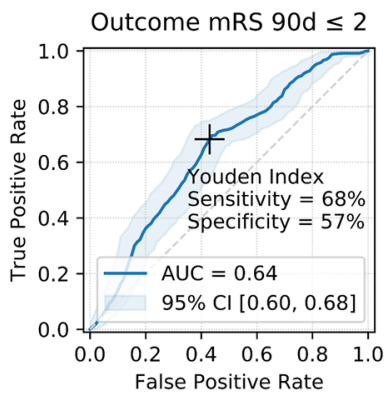

B

Outcome mRS $90 \mathrm{~d} \leq 2$

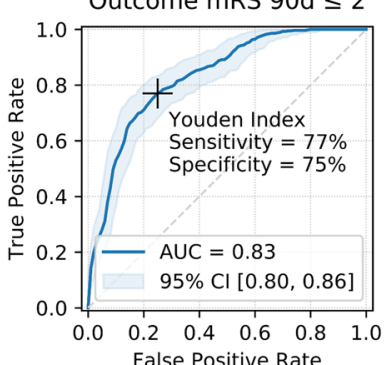

Conventional pc-ASPECTS-based prediction

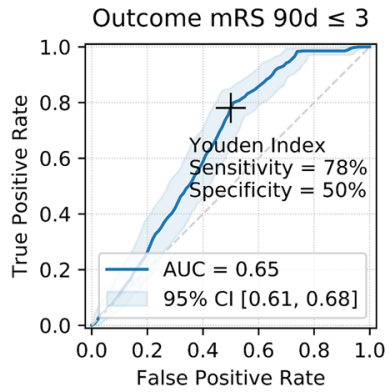

Imaging-based machine-learning prediction

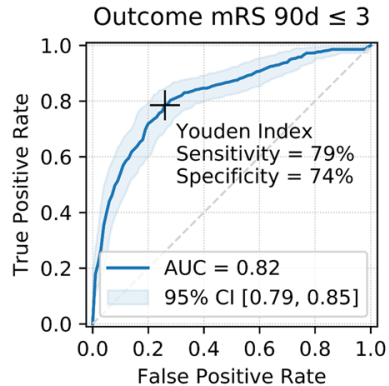

Fig. 2 Imaging-based prediction of outcome in patients with posterior circulation stroke at admission. ROC curves, AUCs and maximum Youden index at different mRS cut-off values for respective binary classification tasks. A Univariate logistic regression models employing conventional pc-ASPECTS ratings; B pure imaging-based random forest machine learning algorithms. Results are based on nested
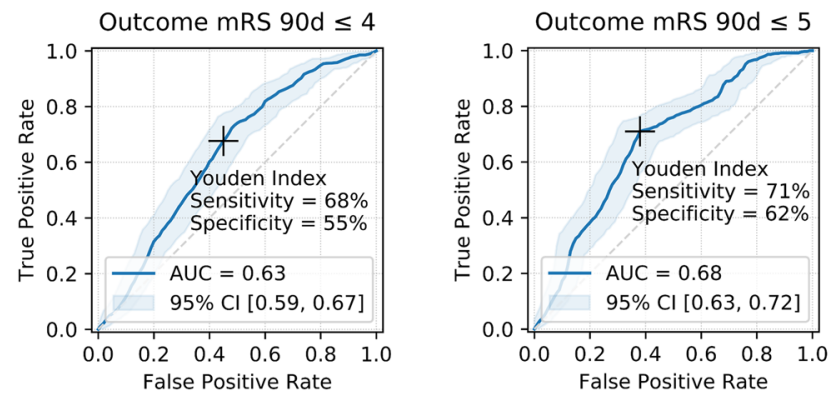

Outcome mRS $90 d \leq 4$

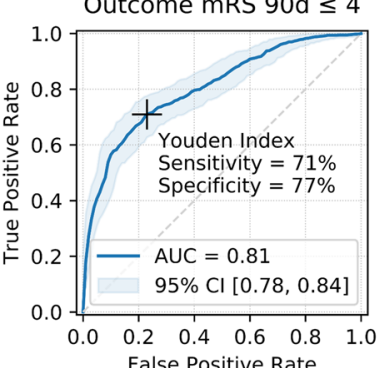

Outcome mRS 90d $\leq 5$

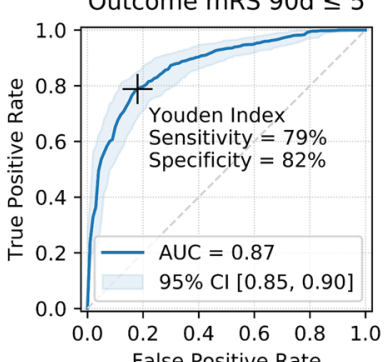

5-fold cross validation of 172 patients from 2 different centers. Bonferroni corrections have been applied to account for alpha spending error. $C I$ confidence interval, $d$ days, $m R S$ modified Rankin Scale, pc-ASPECTS posterior circulation Acute Stroke Prognosis Early CT Score, $R O C A U C$ receiver-operating-characteristic area-under-thecurve 

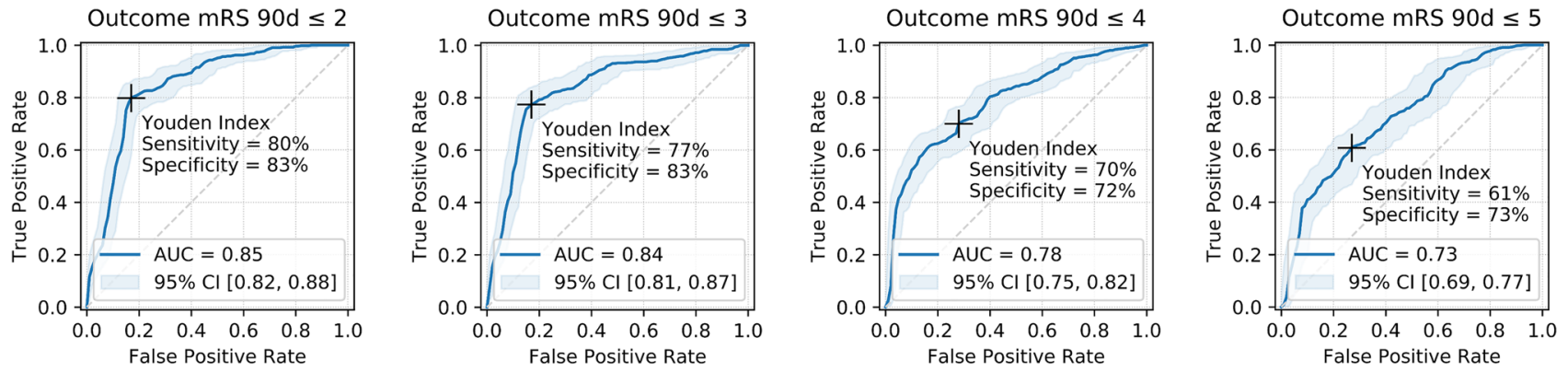

B Conventional pc-ASPECTS, age, NIHSS at admission and imaging-based machine learning prediction
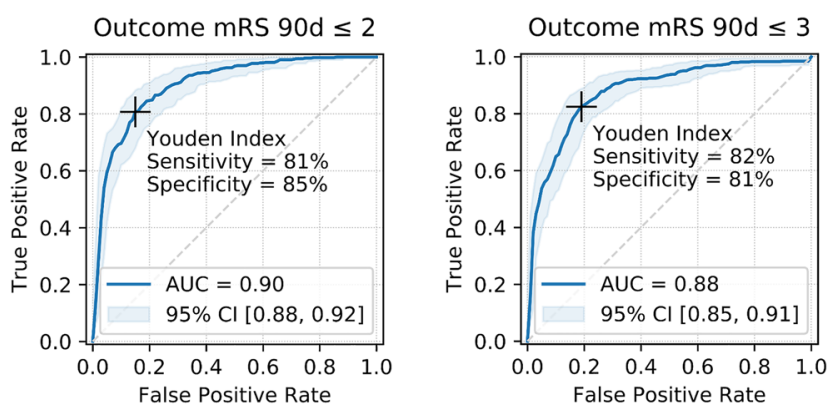

Fig. 3 Imaging and clinical data-based prediction of outcome in patients with posterior circulation stroke at admission. ROC curves, AUCs and maximum Youden index at different mRS cut-off values for respective binary classification tasks. A Multivariate logistic regression models employing conventional predictors of outcome (conventional pc-ASPECTS ratings, NIHSS at admission, age) and B Combined models utilizing information derived from conventional predictors and machine learning-based image analysis. Bonferroni

combined with additional clinical data (NIHSS at admission and age), the conventional prediction model achieved slightly better metrics for differentiating lower mRS values ( $\leq 2$ and $\leq 3$ ), however, these differences were not significant. For the mRS $\leq 4$ and $\leq 5$ classification tasks, quantitative image features-based algorithms reached higher performance with significant differences in all metrics for $\mathrm{mRS} \leq 5$ (survival). The integrated model employing information from conventional pc-ASPECTS ratings, clinical data and machine learning-based evaluation of quantitative image features showed superior results versus conventional pc-ASPECTS and clinical data by trend for all mRS cutoffs. Improvements were observed for ROC AUC in $\mathrm{mRS} \leq 2$ prediction ( $\mathrm{ROC} \mathrm{AUC}=0.90$ vs. $0.85, p$ value $<0.05$ ) and for all metrics in $\mathrm{mRS} \leq 5$ prediction (ROC $\mathrm{AUC}=0.83 \mathrm{vs}$. $0.73, p$ value $<0.05)$. Feature importance analyses of the mean top 300 predictors of all training data sets show that pc-ASPECTS regions with the highest predictive power are cerebellum (30\%), midbrain (29\%) and thalamus (27\%). The largest share of predictive value was mainly derived from wavelet (40\%) and log-sigma (38\%) filtered images. Unfiltered original images contributed $22 \%$ to total predictive
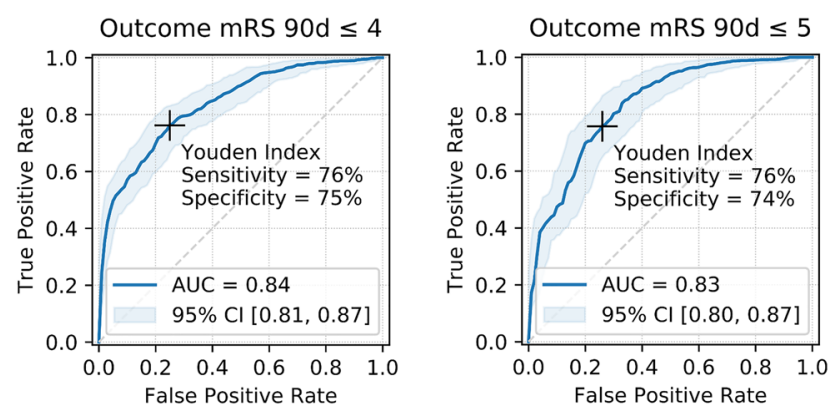

corrections have been applied to account for alpha spending error. Results are based on nested five-fold cross validation of 149 patients from two different centers. $C I$ confidence interval, $d$ days, $m R S$ modified Rankin Scale, NIHSS National Institutes of Health Stroke Scale, $p c$-ASPECTS posterior circulation Acute Stroke Prognosis Early CT Score, $R O C A U C$ receiver-operating-characteristic area under the curve

power (Fig. 4). Within feature classes, texture metrics and first order statistics were used at equal proportions.

\section{Discussion}

In this study, we developed a machine learning approach for predicting functional outcome of patients with posterior circulation stroke based on multidimensional quantitative image analysis of pc-ASPECTS regions in admission NCCTs and basic clinical data available at admission. The study is based on a cohort of 172 patients, of which 57 (33.1\%) achieved a favorable outcome of $m R S \leq 2$ at day 90. This corresponds to the results of the BASIC trial with a recently reported total share of $32.7 \%$ for $\mathrm{mRS} \leq 2$ at day $90(35.1 \%$ in the intervention arm vs. $30.1 \%$ in the control arm) in patients with basilar artery occlusions [32].

Conventional logistic regression and cut-off point optimization confirmed that high pc-ASPECTS (optimal cutoff at pc-ASPECTS $\geq 8$ ) and low NIHSS at admission (optimal cut-off at NIHSS $<10$ ) are significant and independent 
Table 3 Classification performance of imaging-based outcome prediction

\begin{tabular}{|c|c|c|c|c|c|c|}
\hline Prediction mRS & Patients (n; \%) & Classifier model & ROC AUC $[95 \% \mathrm{CI}]$ & $\begin{array}{l}\text { MCC maximum } \\
{[95 \% \mathrm{CI}]}\end{array}$ & $\begin{array}{l}\text { Youden index }[95 \% \\
\text { CI] }\end{array}$ & Accuracy $[95 \% \mathrm{CI}]$ \\
\hline \multirow[t]{4}{*}{$\mathrm{mRS} \leq 2$} & $57 / 172(33 \%)$ & $\begin{array}{l}\text { pc-ASPECTS log } \\
\text { reg }\end{array}$ & $0.64[0.60 ; 0.68]$ & $0.21[0.18 ; 0.24]$ & $26 \%[18 \% ; 33 \%]$ & $65 \%[62 \% ; 68 \%]$ \\
\hline & $57 / 172(33 \%)$ & Img-based ML & $0.83[0.80 ; 0.86]$ & $0.52[0.47 ; 0.58]$ & $52 \%$ [46\%; 58\%] & $76 \%[73 \% ; 79 \%]$ \\
\hline & $53 / 149(31 \%)$ & $\begin{array}{l}\text { pc-ASPECTS and } \\
\text { clin. data log reg }\end{array}$ & $0.85[0.82 ; 0.88]$ & $0.62[0.57 ; 0.66]$ & $63 \%$ [57\%; 69\%] & $82 \%[79 \% ; 85 \%]$ \\
\hline & $53 / 149(31 \%)$ & $\begin{array}{l}\text { Img-based and clin. } \\
\text { data ML }\end{array}$ & $0.90 *[0.88 ; 0.92]$ & $0.65[0.60 ; 0.69]$ & $66 \%[60 \% ; 72 \%]$ & $83 \%[81 \% ; 86 \%]$ \\
\hline \multirow[t]{4}{*}{$\mathrm{mRS} \leq 3$} & $69 / 172(40 \%)$ & $\begin{array}{l}\text { pc-ASPECTS log } \\
\text { reg }\end{array}$ & $0.65[0.61 ; 0.68]$ & $0.36[0.23 ; 0.30]$ & $29 \%[23 \% ; 35 \%]$ & $62 \%[58 \% ; 65 \%]$ \\
\hline & $69 / 172(40 \%)$ & Img-based ML & $0.82[0.79 ; 0.85]$ & $0.52[0.46 ; 0.56]$ & $52 \%[47 \% ; 58 \%]$ & $76 \%[73 \% ; 79 \%]$ \\
\hline & $65 / 149(38 \%)$ & $\begin{array}{l}\text { pc-ASPECTS and } \\
\text { clin. data log reg }\end{array}$ & $0.84[0.81 ; 0.87]$ & $0.60[0.55 ; 0.65]$ & $60 \%$ [54\%; 66\%] & $80 \%[77 \% ; 83 \%]$ \\
\hline & $65 / 149(38 \%)$ & $\begin{array}{l}\text { Img-based and clin. } \\
\text { data ML }\end{array}$ & $0.88[0.85 ; 0.91]$ & $0.63[0.59 ; 0.67]$ & $64 \%$ [58\%; 69\%] & $82 \%[79 \% ; 84 \%]$ \\
\hline \multirow[t]{4}{*}{$\mathrm{mRS} \leq 4$} & $96 / 172(56 \%)$ & $\begin{array}{l}\text { pc-ASPECTS log } \\
\text { reg }\end{array}$ & $0.63[0.59 ; 0.67]$ & $0.25[0.18 ; 0.31]$ & $23 \%[16 \% ; 29 \%]$ & $62 \%[59 \% ; 65 \%]$ \\
\hline & $96 / 172(56 \%)$ & Img-based ML & $0.81[0.78 ; 0.84]$ & $0.48[0.42 ; 0.53]$ & $48 \%[42 \% ; 54 \%]$ & $74 \%$ [71\%; 77\%] \\
\hline & $87 / 149(51 \%)$ & $\begin{array}{l}\text { pc-ASPECTS and } \\
\text { clin. data log reg }\end{array}$ & $0.78[0.75 ; 0.81]$ & $0.41[0.35 ; 0.46]$ & $42 \%$ [35\%; 48\%] & $71 \%[67 \% ; 74 \%]$ \\
\hline & $87 / 149(51 \%)$ & $\begin{array}{l}\text { Img-based and clin. } \\
\text { data ML }\end{array}$ & $0.84 *[0.81 ; 0.87]$ & $0.51 *[0.46 ; 0.56]$ & $51 \%[45 \% ; 57 \%]$ & $75 \%[72 \% ; 78 \%]$ \\
\hline \multirow[t]{4}{*}{$\mathrm{mRS} \leq 5$} & $127 / 172(74 \%)$ & $\begin{array}{l}\text { pc-ASPECTS log } \\
\text { reg }\end{array}$ & $0.68[0.63 ; 0.72]$ & $0.3[0.24 ; 0.36]$ & $33 \%$ [26\%; 40\%] & $69 \%[65 \% ; 72 \%]$ \\
\hline & $127 / 172(74 \%)$ & Img-based ML & $0.87 \dagger[0.85 ; 0.90]$ & $0.56 \dagger[0.51 ; 0.60]$ & $61 \%+[55 \% ; 67 \%]$ & $80 \%+[77 \% ; 82 \%]$ \\
\hline & $115 / 149(67 \%)$ & $\begin{array}{l}\text { pc-ASPECTS and } \\
\text { clin. data log reg }\end{array}$ & $0.73[0.69 ; 0.77]$ & $0.28[0.21 ; 0.35]$ & $34 \%$ [26\%; 41\%] & $64 \%[60 \% ; 67 \%]$ \\
\hline & $115 / 149(67 \%)$ & $\begin{array}{l}\text { Img-based and clin. } \\
\text { data ML }\end{array}$ & $0.83 *[0.80 ; 0.87]$ & $0.49 *[0.43 ; 0.54]$ & $50 \% *[42 \% ; 57 \%]$ & $75 \% *[72 \% ; 78 \%]$ \\
\hline
\end{tabular}

Prediction of outcome in patients with posterior circulation stroke at admission: mRS cut-off values for classification tasks, number of patients with respective outcome (positive class) and performance metrics of logistic regression models employing conventional pc-ASPETCS ratings, pure imaging-based machine learning algorithms, multivariate logistic regression models employing conventional predictors of outcome (conventional pc-ASPECTS ratings, NIHSS at admission, age) and combined models utilizing information derived from conventional predictors and machine learning-based image analysis. Metrics are shown at Youden index maximum cut-off points. Results are based on nested fivefold cross validation of 172 (149) patients from two different centers. Bonferroni corrections have been applied to account for alpha spending error

$C I$ confidence interval, $M C C$ Matthews correlation coefficient, $m R S$ modified Rankin Scale, $p c-A S P E C T S$ posterior circulation Acute Stroke Prognosis Early CT Score, ROC AUC receiver-operating-characteristic area under the curve

${ }^{*} p$ value combined model vs. pc-ASPECTS and clinical data model $<0.05$

${ }^{\dagger} p$ value imaging-based machine learning vs. pc-ASPECTS and clinical data model $<0.05$

predictors of good outcome. These results are in line with the findings of other studies $[2,12]$.

The proposed ML-approach employing quantitative image features provided high discriminatory accuracy between good and poor functional outcome at different $\mathrm{mRS}$ thresholds; observed performance metrics were superior or equal to conventional clinical and imaging-based assessments. For predicting $\mathrm{mRS} \leq 2$, ROC AUC, sensitivity and specificity were $0.90,81 \%$ and $85 \%$ for the integrated machine learning classifier; $0.85,80 \%$ and $83 \%$ for conventional pc-ASPECTS with clinical data and $0.64,68 \%$ and $57 \%$ for conventional pc-ASPECTS alone. Our analysis also showed that employing multidimensional clinical predictors (conventional pc-ASPECTS, NIHSS at admission and age) improved accuracy with a statistically significant increase in ROC AUC compared to using conventional pc-ASPECTS alone. However, even the solely imaging-based machine learning approach achieved higher discriminatory power than conventional pc-ASPECTS.

Earlier studies have investigated the predictive power of conventional pc-ASPECTS ratings based on different imaging modalities and clinical parameters $[2,16,19,21$, 30]. Lin et al. [16] report ROC AUC for pc-ASPECTS and NIHSS at admission of 0.69 and of 0.77 if both parameters are combined. Other works focused on outcome prediction after endovascular therapy and show ROC AUC of 0.74 


\section{Regional feature importance}

\section{Applied filters}
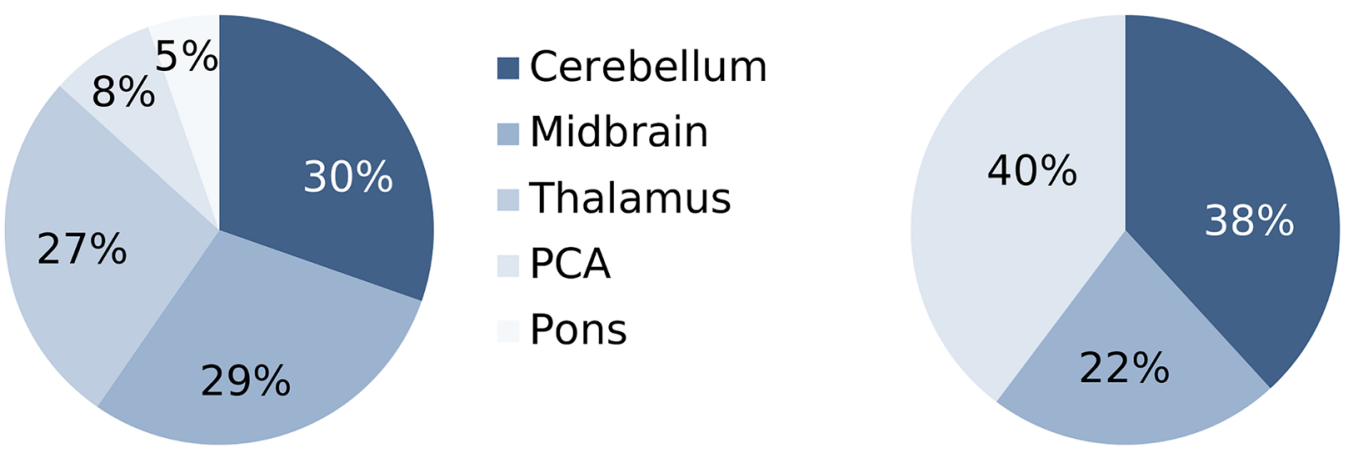

- Log-sigma - Original Wavelet

Fig. 4 Predictive value of quantitative image features. Pie charts show regional distribution of features and applied filters in utilized top-300 predictors. Results are based on nested five-fold cross validation of 172 patients from two different centers. PCA: Posterior cerebral artery

for NIHSS at admission and 0.72 for pc-ASPECTS [21]. Based on CT perfusion imaging parameters, pc-ASPECTS ROC AUC was reported to achieve 0.64 (mean transit time) to 0.82 (cerebral blood volume) [2]. pc-ASPECTS based on DWI was shown to be a predictor of clinical outcome with ROC AUC of 0.82 [19]. To date, all published studies employ conventional regression analysis. None of the published analyses investigated the discriminatory power of ML-based quantitative image assessment in a train, validation and test approach.

Our study has the following limitations: first, generalizability might be limited due to the retrospective nature with inherent selection bias and its relatively small sample size. An expansion of sample size in a prospective study design would certainly contribute to further improving generalizability of results. However, low variability of results across different validation sets suggests sufficient robustness for assessing general feasibility and limitations of the approach. Second, differences in recanalization treatment possibly have influenced patients' outcome. However, our results were confirmed throughout the whole patient collective despite different recanalization results even though our approach did include only variables available at admission. This observation indicates that clinical and imaging data at admission might already include information regarding probabilities of specific treatment strategies (e.g., decision for mechanical thrombectomy based on age, NIHSS, pc-ASPECTS). Furthermore, our findings are supported by the results of the BASICS trial that did not report a significant difference in functional outcome for patients treated with endovascular therapy plus best medical management vs. best medical management alone [32]. Third, limitations typically associated with quantitative radiomics-based image analysis and classification may compromise generalizability of the results $[1,5,6,11,13]$.
These limitations include differences in image acquisition settings, for example size of the field of view or gantry tilt, and under- or overfitting of machine learning algorithms. Bias of these factors was minimized through employment of standardized NCCT scans and the application of Random Forest algorithms that are comparably stable with regards to overfitting. The risk of overfitting was also reduced by evaluating multiple different models in a nested cross-validation approach. Due to standardized and calibrated quantitative imaging parameters and signal intensity processing of CT scanners we assume neglectable bias on classifier performance in a generalized setting. Fourth, with NCCT being the most widely performed brain-imaging technique in acute pc stroke settings [9], our analysis did not integrate CT angiography, CT perfusion or MR imaging. An extension to these imaging modalities could have further improved the results [22, 26-28] as NCCT images only offer limited sensitivity for detecting ischemia compared to e.g. diffusion-weighted imaging [34]. However, both scores-conventional ASPECTS (Alberta Stroke Program Early CT Score) for anterior circulation and pc-ASPECTS - are originally based on evaluations of acute NCCT scans. NCCT scans at admission are fast and the technique is available in most hospitals. Furthermore, NCCT imaging is a fundamental part of most standardof-care stroke protocols. Fifth, the acquisition resolution of NCCT scans was limited to $<5 \mathrm{~mm}$ in slice thickness. The utilization of higher resolution images could improve classification performance. Sixth, the manual definition of pc-ASPECTS areas still implies a certain degree of observer-dependence within the machine learning process. To minimize its influence, we derived standard maps from delineations obtained from 63 healthy subjects. Further, it was shown that radiomic features are comparably stable with regards to variations in segmentations [23, 37]. 


\section{Conclusion}

We developed a machine-learning based classifier that predicts functional outcome of acute posterior circulation stroke patients based on quantitative multidimensional analysis of pc-ASPECTS regions. We observed higher classification performance metrics than achieved in conventional clinical and imaging-based assessments. The proposed algorithm might therefore (1) allow for an early prognosis of the patient's long-term functional status (2) optimize triage for additional MR imaging diagnostics and allocation of best possible medical care [36] and (3) could facilitate required arrangements of the patient's social environment at an early point of time.

Funding Open Access funding enabled and organized by Projekt DEAL. No funding was received towards this work.

\section{Declarations}

Conflicts of interest J. Fiehler is a consultant for Acandis, Boehringer Ingelheim, Codman, Microvention, Sequent, Stryker; a speaker for Bayer Healthcare, Bracco, Covidien/ev3, Penumbra, Philips, Siemens; received grants from Bundesministerium für Wirtschaft und Energie (BMWi), Bundesministerium für Bildung und Forschung (BMBF), Deutsche Forschungsgemeinschaft (DFG), European Union (EU), Covidien, Stryker (THRILL study), Microvention (ERASER study), Philips. G. Thomalla received reports grant support and lecture fees from Bayer, personal fees from Acandis, Boehringer Ingelheim, Bristol-Myers Squibb/Pfizer, and Daiichi Sankyo, Portola, and Stryker. All other authors report no disclosures.

Ethical standards This multi-center retrospective study was approved by the Ethics Committee of the University of Hamburg and the Hamburg Chamber of Physicians, Hamburg, Germany, and the Ethics Committee of the University of Muenster and the Westfalian Chamber of Physicians, Muenster, Germany, and written informed consent was waived by the institutional review boards. All study protocols and procedures were conducted in accordance with the Declaration of Helsinki.

Open Access This article is licensed under a Creative Commons Attribution 4.0 International License, which permits use, sharing, adaptation, distribution and reproduction in any medium or format, as long as you give appropriate credit to the original author(s) and the source, provide a link to the Creative Commons licence, and indicate if changes were made. The images or other third party material in this article are included in the article's Creative Commons licence, unless indicated otherwise in a credit line to the material. If material is not included in the article's Creative Commons licence and your intended use is not permitted by statutory regulation or exceeds the permitted use, you will need to obtain permission directly from the copyright holder. To view a copy of this licence, visit http://creativecommons.org/licenses/by/4.0/.

\section{References}

1. Aerts HJ (2016) The potential of radiomic-based phenotyping in precision medicine: a review. JAMA Oncol 2:1636-1642
2. Alemseged F, Shah DG, Bivard A, Kleinig TJ, Yassi N, Diomedi M, Di Giuliano F, Sharma G, Drew R, Yan B, Dowling RJ, Bush S, Sallustio F, Caltagirone C, Mercuri NB, Floris R, Parsons MW, Levi CR, Mitchell PJ, Davis SM, Campbell BC (2019) Cerebral blood volume lesion extent predicts functional outcome in patients with vertebral and basilar artery occlusion. Int J Stroke 14:540-547

3. Beevor CE (1908) The cerebral arterial supply. Brain 30:403-425

4. Breiman L (2001) Random forests. Mach Learn 45:5-32

5. Davatzikos C (2019) Machine learning in neuroimaging: progress and challenges. Neuroimage 197:652-656

6. Gillies RJ, Kinahan PE, Hricak H (2016) Radiomics: images are more than pictures, they are data. Radiology 278:563-577

7. Glass TA, Hennessey PM, Pazdera L, Chang HM, Wityk RJ, Dewitt LD, Pessin MS, Caplan LR (2002) Outcome at 30 days in the New England Medical Center Posterior Circulation Registry. Arch Neurol 59:369-376

8. Hacke W, Zeumer H, Ferbert A, Bruckmann H, del Zoppo GJ (1988) Intra-arterial thrombolytic therapy improves outcome in patients with acute vertebrobasilar occlusive disease. Stroke 19:1216-1222

9. Hwang DY, Silva GS, Furie KL, Greer DM (2012) Comparative sensitivity of computed tomography vs. magnetic resonance imaging for detecting acute posterior fossa infarct. J Emerg Med 42:559-565

10. Kemmling A, Wersching H, Berger K, Knecht S, Groden C, Nölte I (2012) Decomposing the Hounsfield unit. Clin Neuroradiol 22:79-91

11. Kniep HC, Madesta F, Schneider T, Hanning U, Schonfeld MH, Schon G, Fiehler J, Gauer T, Werner R, Gellissen S (2019) Radiomics of brain MRI: utility in prediction of metastatic tumor type. Radiology 290:479-487

12. Kwak HS, Park JS (2020) Mechanical thrombectomy in basilar artery occlusion. Stroke 51:2045-2050

13. Lambin P, Leijenaar RTH, Deist TM, Peerlings J, de Jong EEC, van Timmeren J, Sanduleanu S, Larue R, Even AJG, Jochems A, van Wijk Y, Woodruff H, van Soest J, Lustberg T, Roelofs E, van Elmpt W, Dekker A, Mottaghy FM, Wildberger JE, Walsh S (2017) Radiomics: the bridge between medical imaging and personalized medicine. Nat Rev Clin Oncol 14:749-762

14. Abud DG, Wermer MJH, Remmers MJM, Schneider H, Rueckert CM, de Laat KF, Yoo AJ, van Doormaal PJ, van Es A, Emmer BJ, Michel P, Puetz V, Audebert HJ, Pontes-Neto OM, Vos JA, Kappelle LJ, Algra A, Schonewille WJ (2021) Endovascular therapy for stroke due to basilar-artery occlusion. N Engl J Med 384:1910-1920

15. Limkin EJ, Sun R, Dercle L, Zacharaki EI, Robert C, Reuze S, Schernberg A, Paragios N, Deutsch E, Ferte C (2017) Promises and challenges for the implementation of computational medical imaging (radiomics) in oncology. Ann Oncol 28:1191-1206

16. Lin SF, Chen CI, Hu HH, Bai CH (2018) Predicting functional outcomes of posterior circulation acute ischemic stroke in first 36 h of stroke onset. J Neurol 265:926-932

17. Lindsberg PJ, Mattle HP (2006) Therapy of basilar artery occlusion: a systematic analysis comparing intra-arterial and intravenous thrombolysis. Stroke 37:922-928

18. Louppe G, Wehenkel L, Sutera A, Geurts P (2013) Understanding variable importances in forests of randomized trees. In: Proceedings of the 26th international conference on neural information processing systems. Curran Associates Inc., Lake Tahoe, Nevada, p 431-439

19. Luo G, Mo D, Tong X, Liebeskind DS, Song L, Ma N, Gao F, Sun X, Zhang X, Wang B, Jia B, Fernandez-Escobar A, Miao Z (2018) Factors associated with 90-day outcomes of patients with acute posterior circulation stroke treated by mechanical thrombectomy. World Neurosurg 109:e318-e328 
20. Matthews BW (1975) Comparison of the predicted and observed secondary structure of T4 phage lysozyme. Biochim Biophys Acta Protein Struct 405:442-451

21. Maus V, Kalkan A, Kabbasch C, Abdullayev N, Stetefeld H, Barnikol UB, Liebig T, Dohmen C, Fink GR, Borggrefe J, Mpotsaris A (2019) Mechanical thrombectomy in basilar artery occlusion: presence of bilateral posterior communicating arteries is a predictor of favorable clinical outcome. Clin Neuroradiol 29:153-160

22. Pallesen LP, Gerber J, Dzialowski I, van der Hoeven EJ, Michel P, Pfefferkorn T, Ozdoba C, Kappelle LJ, Wiedemann B, Khomenko A, Algra A, Hill MD, von Kummer R, Demchuk AM, Schonewille WJ, Puetz V, Group BS (2015) Diagnostic and prognostic impact of pc-ASPECTS applied to perfusion CT in the basilar artery international cooperation study. J Neuroimaging 25:384-389

23. Parmar C, Rios Velazquez E, Leijenaar R, Jermoumi M, Carvalho S, Mak RH, Mitra S, Shankar BU, Kikinis R, Haibe-Kains B, Lambin P, Aerts HJ (2014) Robust Radiomics feature quantification using semiautomatic volumetric segmentation. PLoS ONE 9:e102107

24. Pedregosa F, Varoquaux G, Gramfort A, Michel V, Thirion B, Grisel O, Blondel M, Prettenhofer P, Weiss R, Dubourg V, Vanderplas J, Passos A, Cournapeau D, Brucher M, Perrot M, Duchesnay E (2011) Scikit-learn: machine learning in python. J Mach Learn Res 12:2825-2830

25. Powers DMW (2011) Evaluation: from precision, recall and f-measure to roc., informedness, markedness \& correlation. J Mach Learn Technol 2:37-63

26. Puetz V, Khomenko A, Hill MD, Dzialowski I, Michel P, Weimar C, Wijman CA, Mattle H, Muir K, Pfefferkorn T, Tanne D, Engelter S, Szabo K, Algra A, Demchuk AM, Schonewille WJ, Grp BS (2011) Extent of hypoattenuation on CT angiography source images in basilar artery occlusion: prognostic accuracy of posterior circulation ASPECTS and the pons-midbrain score in the basilar artery international cooperation study. Stroke 42:E118-E119

27. Puetz V, Sylaja PN, Coutts SB, Hill MD, Dzialowski I, Mueller P, Becker U, Urban G, O'Reilly C, Barber PA, Sharma P, Goyal M, Gahn G, von Kummer R, Demchuk AM (2008) Extent of hypoattenuation on $\mathrm{CT}$ angiography source images predicts functional outcome in patients with basilar artery occlusion. Stroke 39:2485-2490
28. Puetz V, Sylaja PN, Hill MD, Coutts SB, Dzialowski I, Becker U, Gahn G, von Kummer R, Demchuk AM (2009) CT angiography source images predict final infarct extent in patients with basilar artery occlusion. AJNR Am J Neuroradiol 30:1877-1883

29. Robin X, Turck N, Hainard A, Tiberti N, Lisacek F, Sanchez JC, Muller M (2011) pROC: an open-source package for R and S+ to analyze and compare ROC curves. BMC Bioinform 12:77

30. Sang H, Li F, Yuan J, Liu S, Luo W, Wen C, Zhu Q, Chen W, Lin M, Qi L, Zhong Y, Wang Z, Ling W, Shi Z, Chen H, Liu W, Liu Z, Yao X, Xiong F, Zeng G, Hu X, Dong H, Mao A, Yang G, Huang J, Chen L, Gong Z, Tao J, Liu H, Wu D, Qiu Z, Yang Q, $\mathrm{Zi} \mathrm{W,} \mathrm{Li} \mathrm{F} \mathrm{(2021)} \mathrm{Values} \mathrm{of} \mathrm{baseline} \mathrm{posterior} \mathrm{circulation} \mathrm{acute}$ stroke prognosis early computed tomography score for treatment decision of acute basilar artery occlusion. Stroke 52:811-820

31. Sato S, Toyoda K, Uehara T, Toratani N, Yokota C, Moriwaki H, Naritomi H, Minematsu K (2008) Baseline NIH stroke scale score predicting outcome in anterior and posterior circulation strokes. Neurology 70:2371-2377

32. Schonewille W (2020) A randomized acute stroke trial of endovascular therapy in acute basilar artery occlusion (BASICS). In: ESO-WSO 2020 major clinical trials webinar, May 13th 2020.

33. Sporns P, Schmidt R, Minnerup J, Dziewas R, Kemmling A, Dittrich R, Zoubi T, Heermann P, Cnyrim C, Schwindt W, Heindel W, Niederstadt T, Hanning U (2016) Computed tomography perfusion improves diagnostic accuracy in acute posterior circulation stroke. Cerebrovasc Dis (Basel, Switzerland) 41:242-247

34. Tei H, Uchiyama S, Usui T, Ohara K (2010) Posterior circulation ASPECTS on diffusion-weighted MRI can be a powerful marker for predicting functional outcome. J Neurol 257:767-773

35. van Griethuysen JJM, Fedorov A, Parmar C, Hosny A, Aucoin N, Narayan V, Beets-Tan RGH, Fillion-Robin JC, Pieper S, Aerts $\mathrm{H}$ (2017) Computational radiomics system to decode the radiographic phenotype. Cancer Res 77:e104-e107

36. Werner MF, Lopez-Rueda A, Zarco FX, Blasco J, San Roman L, Amaro S, Carrero E, Valero R, Oleaga L, Macho JM, Bargallo N (2019) Value of Posterior circulation ASPECTS and ponsmidbrain index on non-contrast CT and CT angiography source images in patients with basilar artery occlusion recanalized after mechanical thrombectomy. Radiologia 61:143-152

37. Yip SS, Aerts HJ (2016) Applications and limitations of radiomics. Phys Med Biol 61:R150-166 